\title{
Grammatical structure and recall: A function of the space in immediate memory or of recall delay?
}

\author{
SAM GLUCKSBERG ${ }^{2}$ AND JOSEPH H. DANKS ${ }^{3}$ \\ PRINCETON UNIVERSITY
}

\begin{abstract}
Savin and Perchonock suggest that when a sentence and additional unrelated words are presented for immediate recall, the number of words recalled in addition to that sentence represents the space left over in a limited memory store after the sentence and its transformational tag have been stored. They report error data and word-recall data in support of a transformational model of sentence processing. In two replications of that study, error data failed to support the transformational model. Furthermore, the word-recall data could be attributed to differential word-recall delays the more complex the sentence, the longer the delay between word presentation and word recall. These findings, as well as others in the literature, fail to support a transformational interpretation of sentence processing.
\end{abstract}

Savin and Perchonock (1965) proposed that the amount of memory space required for remembering a sentence could be estimated by measuring the amount of material that could be recalled in addition to that sentence. They argue that sentences are stored in immediate memory according to some properties described by a transformational grammar (Mehler, 1963; Miller, 1962). Syntactic properties such as negativity and passivity are encoded independently of other properties, and these syntactic "markers" take a certain portion of immediate memory space. Hence, a negative-passive sentence is encoded as a kernel plus two markers, one each for negative and passive, while an active sentence would be encoded as a kernel with no additional markers. ${ }^{4}$ Therefore, the kernel sentence would take up less space in the limited memory store, and leave more room for additional, unrelated material. It thus follows that more unrelated material would be remembered along with the kernel sentence than with a negative-passive sentence.

Savin and Perchonock tested this hypothesis in an ingenious way. On any given trial, $S$ heard a sentence that was followed, after a 5 -sec pause, by a list of eight unrelated words. The S's task was to recall the sentence verbatim, and then to recall as many of the eight additional words as possible. Their findings were consistent with their argument. The number of additional words recalled varied consistently as a function of the syntactic form of the sentences.

An alternative interpretation of these data is possible. Sentences varying in syntactic form may also vary in terms of the time needed to recall and report them. Thus, differential word recall as a function of syntactic form may be attributable to differential word-recall delays, i.e., the time intervals between presentation and recall of the additional words. Several findings lend credence to this interpretation. McMahon (1963) has found that the time needed to decide whether or not a particular sentence is true or false is a function of the syntactic form of that sentence. Similarly, and perhaps more to the point, Johnson (1966) reports that the latency to produce sentences as response terms in a paired-associate list varies as a function of syntactic form. Finally, Matthews (1968), in attempting to replicate the Savin and Perchonock study, found no effect of syntactic form upon recall of unrelated words, but did find that word-recall performance was impaired when a 5-sec delay was imposed upon word recall. These findings suggest that Savin and Perchonock's results may be attributable to differential word-recall delays, and not to the hypothesized space taken up in memory by transformational markers.

Two related hypotheses were tested. The first is that sentence-recall delay, or sentence latency (SL), is a function of the syntactic form of the sentence to be recalled. The greater the number of transformations, the longer will SL be. If this hypothesis holds, a transformational model of sentence processing would be supported. The second hypothesis is that the number of additional words recalled with a sentence is a function of word-recall delay, or word latency (WL), and furthermore that WL is a function of the syntactic form of the sentence. Essentially, this hypothesis assumes that when we vary syntactic form, we simultaneously vary WL, producing a natural analog to Matthew's procedure, in which WL is manipulated directly by instructing Ss to delay their word-recall report.

Experiment 1 is a replication of the Savin and Perchonock study with the addition of two measures: the latency to begin report of the stimulus sentence, and the latency to begin report of the additional words.

\section{EXPERIMENT 1}

\section{Method}

Subjects and experimenter. Twenty undergraduate students at Princeton University served as paid volunteer $S$ s $T$ The $F$ was a trained undergraduate psychology student who was not informed of the experimental hypotheses, and who was unfamiliar with the general problem area. This $\mathrm{E}$ had had a year's experience in data collection in our laboratory and was paid for his time.

Stimulus materials and apparatus. The set of 44 kernel sentences (see Note 4) was identical to the set used by Savin and Perchonock. Each sentence was one that might occur in familiar situations and each contained an animate subject and a present-perfect transitive verb. Table 1 presents an example of one of these kernels with its 10 related transformations.

Four lists of 60 sentences (trials) were constructed. Each list had 10 kernels and five sentences in each of the other 10 transformations. The sentences were arranged in random order. Each of the 44 sentences was used no more than six times in all four lists and no more than twice in any given list (and then in two different transformational forms). Any particular form of a sentence was used only once across the four lists. The subsets of unrelated words following each sentence were the same for any given trial in all lists. Five Ss were assigned to each of the four lists.

The four lists were tape-recorded by one of the authors (JHD). The sentences were spoken with an even intonation, except for the sentences with the emphatic transformation, and the words were spoken at the rate of $1 / 0.75 \mathrm{sec}$. After all stimuli had been recorded, the naive $E$ placed an inaudible signal on a second track of the recording tape at the end of each word list. This signal started two timers simultaneously. One of these timers was stopped by a voice key activated by $S$ when he began his verbatim recall of the stimulus sentence, providing a measure of sentence-recall latency (SL). The second timer was stopped manually by $E$ when $S$ uttered the first word of the word list, 
Table 1

Sample of Stimulus Materials Used

\begin{tabular}{|c|c|c|}
\hline $\begin{array}{l}\text { Number of Transformations } \\
\text { (T) }\end{array}$ & Sentence Type & Example \\
\hline 0 & Kernel (K) & The woman has bought the dress. \\
\hline 1 & $\begin{array}{l}\text { Passive (P) } \\
\text { Question (Q) } \\
\text { Negative (N) } \\
\text { *Question-Negative (QN) } \\
\text { Emphatic (E) } \\
\text { Wh-Question (Wh-) }\end{array}$ & $\begin{array}{l}\text { The dress has been bought by the woman. } \\
\text { Has the woman bought the dress? } \\
\text { The woman has not bought the dress. } \\
\text { Has not the woman bought the dress? } \\
\text { The woman has bought the dress. } \\
\text { Who has bought the dress? }\end{array}$ \\
\hline 2 & $\begin{array}{l}\text { Passive-Question (PQ) } \\
\text { Passive-Negative (PN) } \\
\text { *Passive-Question-Negative (PQN) } \\
\text { Passive-Emphatic (PE) }\end{array}$ & $\begin{array}{l}\text { Has the dress been bought by the woman? } \\
\text { The dress has not been bought by the woman. } \\
\text { Has the dress not been bought by the woman? } \\
\text { The dress has been bought by the woman. }\end{array}$ \\
\hline
\end{tabular}

* As did Savin and Perchonock, we consider the $Q_{N}$ sentence type to represent a single transformation, equivalent to $P, Q, N, E$, and Wh-. Katz and Postal (1964) present formal reasons for this interpretation, and Clifton and Odom (1966) provide convincing evidence that such sentences are perceived in this way by native English speakers.

providing a measure of word-recall latency (WL). WL thus served as a measure of the delay between the end of the word-list presentation and the beginning of word recall, analogous to the retention interval in a short-term memory paradigm.

Procedure. Savin and Perchonock's procedure was followed as closely as possible. The $\mathrm{S}$ was told that he would hear a sentence followed by a brief pause $(5 \mathrm{sec})$ and then by a list of eight words. A card with a list of word categories was provided to aid recall, and the possible words in each category were read aloud to him. The order of categories was constant for all trials. The $\mathrm{S}$ was to recall the sentence verbatim and then as many of the additional words as possible. The Ss were free to request rest breaks any time they felt it would improve their performance. Typically, one such break was requested during the 60-trial session.

\section{Results and Discussion}

Sentence-recall errors. Eighty-five sentences $(7.1 \%$ of the total number) were recalled with transformational errors only. Following Savin and Perchonock's procedure, these sentences were treated in terms of the transformational form of the response. We assumed that these errors were primarily listening errors, and this assumption is supported by the observation that $66(78 \%)$ of these errors were deletions of the emphatic transformation, a transformation that involves a stress pattern and could easily be heard incorrectly. The remaining 19 transformational errors were distributed evenly over the sentence types. Sixty sentences had other types of errors, and 10 of these also had transformational errors. Of these 60,36 were trivial, involving change of tense, synonym substitution, and the like. Twenty-four were serious enough to justify deleting them from the word-recall data, though they were retained for the analysis of sentence errors. These consisted of trials in which words, phrases, or the entire sentence were omitted or were grossly different from the stimulus sentence.

These error data are comparable to those of Savin and Perchonock with one notable exception. One of their principal assumptions was that transformations are stored independently of one another and of the rest of the sentence. The pattern of errors that we obtained is not consistent with this view. About $7.1 \%$ of sentence responses contained transformational errors, and 5.0\% contained other kinds of errors. If transformational tags are remembered independently of other information about the sentence, then we would expect only $0.35 \%$ of the sentence responses to contain both types of errors $(.071 \times .050=.0035)$. In fact, there were 10 such errors $(0.83 \%)$, more than double the expected number of occurrences. These data suggest that transformational errors are not independent of other types of errors.

Sentence-recall latency. If SL is influenced by the theoretical number of transformational tags involved, then ordinal predictions can be made between any two sentence types, $\mathbf{A}$ and $B$, if one set of such tags, $a$, is a proper subset of the other set, $b$. It should take longer to process $B$ because more information is involved. If one set is not a proper subset of the other, then no prediction can be made. For example, predictions can be made about differences between $K, P$, and $P Q$, but not about differences between $P$ and $Q$. The possible predictions are:

$$
\begin{aligned}
& \mathrm{K}<\mathrm{N}, \mathrm{P}, \mathrm{Q}, \mathrm{E}, \mathrm{PN}, \mathrm{Q}_{\mathrm{N}}, \mathrm{PQ}, \mathrm{EP}, \mathrm{PQ}_{\mathrm{N}} \\
& \mathrm{N}<\mathrm{NP} \\
& \mathrm{P}<\mathrm{PQ}, \mathrm{PQ}_{\mathrm{N}}, \mathrm{PE} \\
& \mathrm{Q}<\mathrm{PQ} \\
& \mathrm{E}<\mathrm{PE} \\
& \mathrm{Q}_{\mathrm{N}}<\mathrm{PQ}_{\mathrm{N}}
\end{aligned}
$$

These predictions concerning SL are exactly parallel to the predictions made by Savin and Perchonock concerning the number of words recalled with each sentence type. Table 2 presents the SL data obtained, together with other data of interest: WL and mean words recalled (WR). Of the $17 \mathrm{SL}$ differences predicted by the transformational hypothesis, none was reliable at the .05 level (two-tailed sign tests).

In order to provide a less stringent test of the transformational hypothesis concerning SL, the data were grouped into three categories depending upon the number of hypothetical transformational tags each sentence had. Thus, the kernel would have zero tags, single transform sentences, such as $\mathrm{N}$, one tag, and double transform sentences, such as NP, two tags. Each sentence used was thus pooled with all other sentences having the same number of tags (T). Table 3 presents these pooled data. A Ss by Treatments analysis of variance was applied to the SL data. No effect of syntax (T) on SL was obtained, although the differences between the means were in the predicted directions, $F(2,38)=1.41, p>.05$. Sentence latency, then, does not seem to be affected by the syntactic form of the sentence.

Word recall. Mean WR as a function of sentence type is presented in Table 2 . The ordinal predictions are precisely parallel to the predictions concerning SL. Of the 17 predicted differences, only 2 ( $\mathrm{K}>\mathrm{PQ}, \mathrm{PN}$ ) approached the .05 level, $\mathrm{p}<.06$, two-tailed sign tests. One difference $(P Q>Q)$ was reliable in the wrong direction, $\mathrm{p}=.04$, two-tailed sign test.

The WR data were grouped as the SL data were, in terms of the $T$ value of the sentences, and the appropriate analysis was 
Table 2

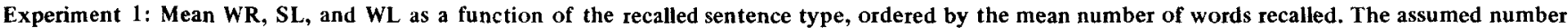
of transformations are indicated in parentheses

\begin{tabular}{|c|c|c|c|c|c|c|c|c|c|c|c|}
\hline & \multicolumn{11}{|c|}{ Sentence Type } \\
\hline & Wh- (1) & $\mathrm{K}(0)$ & $\mathrm{QN}_{\mathrm{N}}(1)$ & $\mathrm{E}(1)$ & $\mathrm{N}(1)$ & PE (2) & $\mathrm{PQ}(2)$ & PQN (2) & $P(1)$ & $\mathrm{Q}(1)$ & $\mathrm{PN}$ (2) \\
\hline WR (words) & 4.98 & 4.88 & 4.87 & 4.86 & 4.81 & 4.80 & 4.62 & 4.51 & 4.47 & 4.45 & 4.40 \\
\hline SL (sec) & 1.60 & 1.23 & 1.26 & 1.33 & 1.28 & 1.06 & 1.66 & 1.27 & 1.51 & 1.30 & 1.55 \\
\hline WL (sec) & 4.40 & 4.22 & 4.48 & 4.58 & 4.45 & 4.62 & 5.38 & 4.83 & 5.11 & 4.41 & 5.20 \\
\hline
\end{tabular}

computed (see Table 3). A significant effect of $T$ upon WR was obtained, $F(2,38)=7.50, p<.01$. Word recall does seem to be a function of syntactic form, but the effect obtained here is much weaker than that obtained by Savin and Perchonock. We turn now to the word-latency data to see if the differential word recall obtained is paralleled by differential word latencies as a function of $\mathrm{T}$.

Word recall and word latency. The WL data were pooled in terms of $T$ and analyzed in the same way that the SL and WR data were (see Table 3). As with the WR data, a significant effect of $\mathrm{T}$ was obtained, $\mathrm{F}(2,38)=20.22, \mathrm{p}<.001$.

In order to evaluate the relation between WR and WL, an Ss by Treatments analysis of variance was applied to the WR data as a function of WL. The treatment levels were the means of five 12-trial blocks for each $S$ after the trials had been rank-ordered on the basis of each S's WL scores. Mean WR as a function of WL is presented in Fig. 1. As expected, WR declines as WL increases, and this effect is significant at the .001 level, $F(4,76)=13.13$. These data are consistent with those reported by Matthews: the longer the delay between word presentation and word recall, the poorer the recall.

The main findings of Experiment 1 were (a) the error data did not support a transformational model of sentence processing, and (b) word recall was, to some extent, a function of syntactic form, but the effects of syntactic form could have been mediated by differential word-recall delays, which varied as a function of syntactic form. One possible interpretation of these data is that formal grammatical transformations need not be isomorphic with psychological transformations (Miller \& Chomsky, 1963), nor need they be invariantly complex across samples of people. For example, sentences that begin with the phrase "It is not unlikely that ..." involve, at the formal level, several transformations. Yet, it is not unlikely that most members of the academic community deal with such sentences without psychological transformations. Rather, the sentence is an immediately understood cliché. In this way, formal transformations that may have once required laborious decoding can, through learning, become psychologically simple.

If this is the case, then the more linguistically sophisticated or competent a $S$ population is, the less likely will that population process sentences in a manner analogous to transformational theory. While the Ss of Experiment 1 (Princeton undergraduates) are quite comparable to the Ss employed by Savin and Perchonock (University of Pennsylvania undergraduates), it could still be the case that their results could be achieved more readily

Table 3

Word recall, sentence latency and word-recall latency as a function of $T$.

\begin{tabular}{lllllll}
\hline & & \multicolumn{5}{c}{ T } \\
\hline & Mean & SD & Mean & SD & Mean & SD \\
\hline WR (words) & 4.93 & 0.91 & 4.69 & 0.85 & 4.54 & 0.86 \\
SL (sec) & 1.28 & 0.75 & 1.33 & 0.96 & 1.42 & 1.07 \\
WL (sec) & 4.32 & 1.41 & 4.62 & 1.53 & 5.02 & 1.56 \\
\hline
\end{tabular}

with a younger, less verbally competent group of Ss. We therefore replicated Experiment 1 with high-school-age Ss.

\section{EXPERIMENT 2}

\section{Method}

Twenty male Princeton High School students (mean age $=$ 15.35, range 14-16) served as paid volunteer Ss. Experiment 1 was repeated exactly, with one exception. Half the Ss were tested by the same naive $\mathrm{E}$ who had collected the data in Experiment 1; the remaining $10 \mathrm{Ss}$ were tested by one of the authors (JHD). Assignment to lists and to Es was random, with Es counterbalanced across lists. The only apparent difference in the conduct of Experiments 1 and 2 was that the high-school Ss requested one to two more rest breaks than did the college $S$ s.

\section{Results and Discussion}

Sentence recall errors. The error rate here was slightly higher than in Experiment $1-218$ errors were made (18\%). Transformational errors accounted for 130 of these, and of these 130,100 were deletions of the emphatic transformation. The other 30 transformational errors were evenly distributed across sentence types, indicating no trend towards loss of transformations as a primary source of error. Of the remaining errors, 63 were minor changes of tense or synonym substitutions. Forty were gross distortions or complete sentence omissions. These 40 were omitted from the latency and word-recall analyses. There were no reliable differences in sentence errors as a function of $\mathrm{E}$.

The discrepancy between the obtained error data and those predicted by a transformational model was smaller than in Experiment 1, but was still present. The probability of a transformational error was .108 , and of a nontransformational error, .086 , yielding a predicted joint occurrence of $p=.0093$ if we assume independence. The obtained proportion of joint occurrence was .0125 .

Sentence-recall latency. Of the 17 SL differences predicted (see Experiment 1), one was significant in the right direction $(\mathrm{P}<\mathrm{PN})$ at the .05 level and one was significant in the wrong direction $\left(\mathrm{PQ}_{\mathrm{N}}<\mathrm{P}\right)$ (see Table 4). As in Experiment 1, the data were grouped in terms of $T$, and a $T$ by Experimenter analysis of variance was performed (see Table 5 for group means). Neither of the main effects nor the interaction was significant. As in Experiment 1, sentence form did not affect sentence-recall latency.

Word recall. Of the 17 predicted differences in WR, none was significant (two-tailed sign tests) at the .05 level (see Table 4). When the WR data were grouped (see Table 5) and the appropriate analysis of variance computed, the effect of syntax was significant, $F(2,36)=3.27, p<.05$. The effect of $E$ was also significant, $F(1,18)=8.46, p<.01$, but $E$ did not interact significantly with $\mathrm{T}, \mathrm{F}(2,36)=1.49, \mathrm{p}>.05$. As in Experiment 1 WR did vary as a function of $T$, the number of formal sentence transforms.

Word recall and word latency. The effect of $\mathrm{T}$ upon WL was again significant, $F(2,36)=15.09, p<.001$. There was no main effect of $E, F(1,18)<1.0$, while the interaction of $E$ and $T$ was significant, $F(2,36)=3.63, p<.05$. This interaction reflects a 


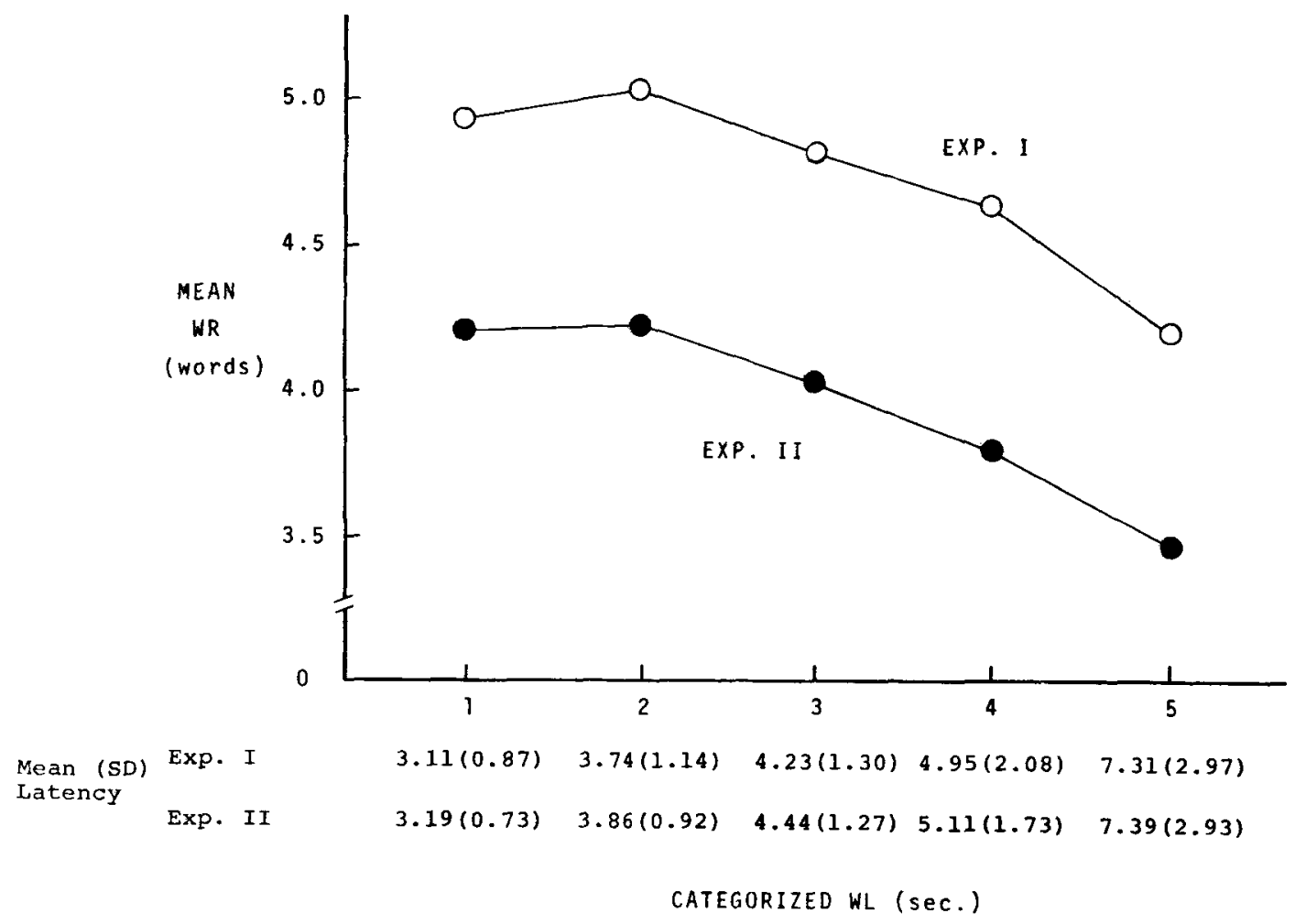

Fig. 1. Experiments 1 and 2: Mean WR as a function of categorized WL-rank ordered from shortest to longest latency.

difference in the extent to which $T$ influenced WL as a function of $E$, but not the direction of the effect.

An analysis of variance similar to that used in Experiment 1 was applied to the Experiment 2 data to test the relationship between WR and WL (see Fig. 1). The main effect of WL was significant at the .001 level, $F(4,72)=9.24$.

In summary, the results of Experiment 2 replicate those of Experiment 1 in every detail. The effects of sentence type were small, and word recall is significantly related to word-recall delay, as measured by word latency.

\section{GENERAL DISCUSSION}

The findings of Savin and Perchonock were, in general, replicated, but alternative interpretations are suggested by the various other findings obtained. First, the error data do not provide consistent support for the transformational model that was originally proposed. Transformational errors were not independent of other errors, and the errors could not be interpreted as losses of transformational tags, or markers. Second, there was a strong relationship between word latency and word recall, both in this study and in the study reported by Matthews (1968). In the Matthews study, word latency was manipulated directly by $\mathrm{E}$. Here, word latency was permitted to vary spontaneously, and the effect was obtained nonetheless. There are at least two possible interpretations of this relationship.

(A) WL and WR may be independent effects of a third variable, T. Transformational tags may indeed be stored as suggested by Savin and Perchonock, and thus T could affect the storage space available for unrelated words. $T$ may also affect WL independently of the effects upon storage space, e.g., sentence length varies directly with $T$. Mean sentence length for 0,1 , and 2 transformations was $6.0,6.6$, and 8.5 words, respectively. The longer the sentence, the longer it may take to report it, and thus WL could vary as a function of sentence length. However, we know of no data relating sentence length to time to utter sentences, and so this is only a possibility.

(B) The second possibility is that the relation between WR and $T$ is attributable entirely to the effects of $T$ upon WL, that is, forgetting in this situation is simply a function of time. While our data do not provide a direct test which would discriminate between (A) and (B), several arguments can be cited in support of the simpler hypothesis, (B).

First, in both experiments, WL and WR were each related to $T$. Ignoring T, WR was a significant function of WL. If (B) represents the true state of affairs, then the product of the proportion of within-Ss variance in WL accounted for by $T$ and the proportion

Table 4

Experiment 2: Mean WR, SL, and WL as a function of the recalled sentence type, ordered by the mean number of words recalled. The assumed number of transformations are indicated in parentheses.

\begin{tabular}{lcccccccccccc}
\hline & \multicolumn{1}{c}{ Sentence Type } \\
\hline WR (words) & K (0) & N (1) & QN (1) & Wh- (1) & E (1) & PQ (2) & Q (1) & P (1) & PQN (2) & PN (2) & PE (2) \\
SL (sec) & 4.06 & 4.05 & 4.03 & 4.02 & 4.00 & 3.89 & 3.86 & 3.76 & 3.75 & 3.71 & 3.60 & 1.71 \\
WL (sec) & 1.22 & 1.33 & 1.05 & 1.15 & 1.23 & 1.34 & 1.23 & 1.28 & 1.05 & 1.45 & 1.25 & 4.25 \\
\hline
\end{tabular}


Table 5

Experiment 2: Word recall, sentence latency and word-recall latency.

\begin{tabular}{lrlllll}
\hline & & & & & & \\
\end{tabular}

of within-Ss variance in WR accounted for by WL should yield, or predict, the proportion of within-Ss variance in WR accounted for by $T$. That is, if:

$$
W L=f_{1}(T)
$$

and $\quad W R=f_{2}(W L)$

then $W_{p}=\left[f_{1}(T) \cdot f_{2}(W L)\right]$

[predicted]

should predict

$$
W_{R_{0}}=f_{3}(T) \quad \text { [obtained] }
$$

where, for $X=f_{i}(Y), f_{i}(Y)$ is the proportion of within-Ss variance in $\mathrm{X}$ accounted for by $\mathrm{Y}$. For the data of Experiment 1, $f_{1}(T)=.484$ and $f_{2}(W L)=.375$. The predicted $W R_{p}$ is .182 , and the obtained $W_{0}$ was .241 . For Experiment 2, $f_{1}(T)=3.79$, $\mathrm{f}_{2}(\mathrm{WL})=.291$, yielding a predicted $\mathrm{WR}_{\mathrm{p}}$ of .110 , compared with an obtained $W_{\mathrm{o}}$ of .098 . These correspondences between the predicted and obtained values, while not perfect, are sufficiently close to lend credence to our argument.

Second, consider some characteristics of immediate and long-term memory. Savin and Perchonock's argument assumes that space in immediate memory is finite and limited. Their argument also makes the assumption that sentences in immediate memory are represented in other than merely acoustic, or rote, form. In view of the data on sources of confusion in immediate memory, it would seem that inmediate memory storage involves primarily acoustic or articulatory modes (Baddeley, 1964). If this is so, then Ss engaged in the task used here may be responding primarily to the surface structure rather than to the deep structure represented by the kernel plus transformational tag(s). Considering the rather rapid pacing of the task, and the emphasized instructions to repeat the sentence exactly, it is not unreasonable to suspect that $S s$ would tend to deal with the material in a rote fashion. Thus, aside from affecting sentence length, $\mathrm{T}$ would not affect performance.

If, on the other hand, one assumes that the sentences were decoded, that is, were transferred from immediate memory into another form in some intermediate or long-term store, then again no direct behavioral effects of $T$ should be expected. Assume that during the 5 -sec pause between the end of a sentence presentation and the beginning of the word-list presentation, Ss process the sentence. Under these circumstances, immediate memory should be cleared, with space now available for word storage. When, at the end of the word list, the sentence must be retrieved, any thing that increases the time needed to report the sentence would affect the subsequent word-recall performance. The most parsimonious interpretation of the word-recall data would thus be in terms of the word-recall latency. Any effects of syntactic form upon word recall is most simply attributable to the relation, for whatever reasons, between syntactic form and word-recall latency.

These arguments do not speak directly to the theoretical model proposed by Savin and Perchonock. They do, however, suggest that their choice of experimental technique is inappropriate for testing that model. Forgetting as a simple function of time provides the most parsimonious explanation of our WR data and may provide the most parsimonious explanation of Savin and Perchonock's findings as well, at least until a relationship between $T$ and WR can be demonstrated which is not confounded with time.

\section{REFERENCES}

BADDELEY, A. S. Semantic and acoustic similarity in short-term memory. Nature, 1964, 204, 1116-1117.

CLIFTON, C., JR., \& ODOM, P. Similarity relations among certain English sentence constructions. Psychological Monographs, 1966, 80 (5, Whole No. 613).

JOHNSON, N. F. On the relationship between sentence structure and the latency in generating the sentence. Journal of Verbal Learning \& Verbal Behavior, 1966, 5, 375-380.

KATZ, J. I., \& POSTAL, P. M. An integrated theory of linguistic descriptions. Cambridge, Mass.: M.I.T., 1964.

MATTHEWS, W. A. Transformational complexity and short-term recall. Language \& Speech, 1968, 11, 120-128.

McMAHON, L. E. Grammatical analysis as part of understanding a sentence. Unpublished doctoral dissertation, Harvard University, 1963.

MEHLER, J. Some effects of grammatical transformations on the recall of English sent nces. Journal of Verbal Learning \& Verbal Behavior, 1963, $2,346-351$.

MILLER, G. A. Some psychological studies of grammar. American Psychologist, 1962, 17, 748-762.

MILLER, G. A., \& CHOMSKY, N. Finitary models of language users. In R. D. Luce, R. R. Bush, and E. Galanter (Eds.), Handbook of mathematical psychology, Vol. 2. New York: Wiley, 1963. Pp. 419-491.

SAVIN, H. B., \& PERCHONOCK, E. Grammatical structure and the immediate recall of English sentences. Journal of Verbal Learning \& Verbal Behavior, 1965, 4, 348-353.

\section{NOTES}

1. Bryant Crouse's assistance in data collection is gratefully acknowledged, and we wish to thank Harris Savin for making his stimulus materials available to us. This work was supported in part by Public Health Service Grants MH10742 and HD01910 to Princeton University. Additional support was provided by a PHS Predoctoral Fellowship to J. H. Danks.

2. Address: Department of Psychology, Princeton University, Princeton, New Jersey 08540 .

3. Now at Kent State University, Kent, Ohio 44240.

4. These sentences are not appropriately termed "kernels"; rather, they should be described as "simple, active, affirmative, declarative." However, to facilitate comparison with Savin and Perchonock, we use their notation.

(Accepted for publication January 27, 1969.) 